\title{
CUIDADOS PALIATIVOS EM UM AMBIENTE HOSPITALAR: UM RELATO DE EXPERIÊNCIA
}

\author{
PALLIATIVE CARE IN A HOSPITAL: AN EXPERIENCE REPORT
}

\section{Laura Fernandes Ferreira ${ }^{1}$}

RESUMO: Estamos diante de uma intensa luta em busca da cura das doenças crônicodegenerativas intratáveis o que levou a uma cultura de negação da morte, sendo encarada como derrota ou fracasso pelos profissionais de saúde. Diante disso os cuidados paliativos surgiram como forma de mudar a definição de "morte" criada á séculos, como uma forma de inovar a assistência na área da saúde, ganhando cada vez mais espaço no Brasil. Este tipo de cuidado foi definido em 2002 pela organização mundial de saúde (OMS), abordando a promoção da qualidade de vida de pacientes e seus familiares, através da avaliação precoce e controle de sintomas físicos, sociais, emocionais, espirituais desagradáveis, em um contexto de doenças que ameaçam a continuidade da vida. Existem mais de 7.000 serviços de cuidados paliativos em mais de 90 países no mundo, sendo no Brasil apenas 40 serviços especializados nessa área. Não existe um lugar específico para programar os cuidados paliativos, o local indicado é onde o paciente estiver, onde necessitar de cuidados. E um grande número dos pacientes e familiares opta por "falecer" nos hospitais, onde os cuidados paliativos devem ser realizados. Dessa forma o objetivo deste estudo consiste em demonstrar a importância de programar cuidados paliativos em um ambiente hospitalar o qual recebe pacientes portadores de múltiplas comorbidades, principalmente comorbidades crônico-degenerativas com limitações no que rege a cura, como forma de garantir uma melhor qualidade de vida aos pacientes e seus familiares.

Palavras-chave: Paliatividade. Cuidados paliativos. Cuidar.

ABSTRACT: We are facing an intense struggle to cure intractable chronic-degenerative diseases, which has led to a culture of denial of death, which is seen as defeat or failure by health professionals. Therefore, palliative care emerged as a way to change the definition of "death" created centuries ago, as a way to innovate healthcare assistance, gaining more and more space in Brazil. This type of care was defined in 2002 by the World Health Organization (WHO), addressing the promotion of the quality of life of patients and their families, through the early assessment and control of unpleasant physical, social, emotional, spiritual symptoms, in a context diseases that threaten the continuity of life. There are more than 7,0oo palliative care services in more than 90 countries worldwide, with only 40 specialized services in this area in Brazil. There is no specific place to schedule palliative care, the indicated place is where the patient is, where he needs care. And a large number of patients and family members choose to "die" in hospitals, where palliative care must be performed. Thus, the objective of this study is to demonstrate the importance of scheduling palliative care in a hospital environment that receives patients with multiple comorbidities, especially chronicdegenerative comorbidities with limitations in what governs the cure, as a way to guarantee a better quality of life. patients and their families.

\footnotetext{
I Formada em medicina pela Faculdade Atenas e residente de Clínica Médica pela Universidade Evangélica de Anápolis- Goiás. E-mail: laurafernandes3@hotmail.com.
} 
Keywords: Palliative care. Palliative care. Take care.

\section{INTRODUÇÃO}

Com os avanços na medicina a esperança de vida vem aumentando cada vez mais, caracterizado pela inversão da pirâmide populacional, e juntamente a isso o número de pacientes com doenças crônico-degenerativas incapacitantes e incuráveis. Dessa forma estamos diante de uma intensa luta em busca da cura dessas doenças o que levou a uma cultura de negação da morte, sendo encarada como derrota ou fracasso pelos profissionais de saúde. Diante disso os cuidados paliativos surgiram como forma de mudar a definição de "morte" criada á séculos, como uma forma de inovar a assistência na área da saúde, ganhando cada vez mais espaço no Brasil. ${ }^{2}$

Este tipo de cuidado foi definido em 2002 pela organização mundial de saúde (OMS), abordando a promoção da qualidade de vida de pacientes e seus familiares, através da avaliação precoce e controle de sintomas físicos, sociais, emocionais, espirituais desagradáveis, em um contexto de doenças que ameaçam a continuidade da vida. ${ }^{5}$

Essa forma de cuidar consiste em aliviar o sofrimento do paciente, garantindo uma qualidade de vida não somente ao indivíduo portador da doença avançada, mas também aos familiares, mantendo uma boa comunicação, controle de dor e outros sintomas, suporte espiritual, psicossocial e no enlutamento. O paciente em cuidados paliativos não é só um ser biológico, ele é também um ser social, espiritual e psicológico e se alguma dessas não funcionam bem, outras são afetadas, sendo assim, para exercer os cuidados, esses são dependentes de uma equipe multiprofissional, para que todos os aspectos sejam cuidados, seja ele físico, mental, espiritual e social. ${ }^{1,3}$. Equipe a qual, segundo o ministério da saúde, deve trabalhar em sintonia de forma a contemplar a autonomia do paciente e de sua família, sendo composta por no mínimo: médicos, enfermeiros, nutricionistas, psicólogos, assistente social, fisioterapeutas e terapeutas ocupacionais. ${ }^{4}$

Para uma adequada prática de cuidados paliativos são necessários conhecimento e compreensão dos seguintes princípios norteadores (MATSUMOTO, 2009) ${ }^{13}$ :

- Iniciar o mais precocemente possível o acompanhamento em cuidados paliativos junto a tratamentos modificadores da doença. Incluir toda a investigação necessária para compreender qual o melhor tratamento e manejo dos sintomas apresentados.

- Reafirmar a vida e sua importância.

- Compreender a morte como processo natural sem antecipar nem postergá-la. 
- Promover avaliação, reavaliação e alívio impecável da dor e de outros sintomas geradores de desconforto.

- Perceber o indivíduo em toda sua completude, incluindo aspectos psicossociais e espirituais no seu cuidado. Para isso é imprescindível uma equipe multidisciplinar.

- Oferecer o melhor suporte ao paciente focando na melhora da qualidade de vida, influenciando positivamente no curso da doença quando houver possibilidade e auxiliando-o a viver tão ativamente quanto possível até a sua morte.

- Compreender os familiares e entes queridos como parte importante do processo, oferecendo-lhes suporte e amparo durante o adoecimento do paciente e também no processo de luto após o óbito do paciente. ${ }^{5}$

Sendo assim o objetivo deste estudo consiste em demonstrar a importância de implementar cuidados paliativos em um ambiente hospitalar o qual recebe pacientes portadores de múltiplas comorbidades, principalmente comorbidades crônico-degenerativas com limitações no que rege a cura, como forma de garantir uma melhor qualidade de vida aos pacientes e seus familiares. Desta forma, participando de um contexto no qual a morte aparece todos os dias, é necessário e essencial que todos os profissionais de saúde da unidade saibam lidar com o medo da morte e o processo de morrer, prestando, assim uma assistência e conforto necessário ao paciente e sua família.

\section{Metodologia}

Este é um estudo descritivo, qualitativo, do tipo relato de experiência que de acordo com Minayo, consiste em um caminho metodológico que permite a descrição de situações vivenciadas pelos autores de uma pesquisa. Trata-se de um relato vivenciado em um Hospital do município de Anápolis, Goiás no ano de 2019 e 2020 durante o período de residência médica na área de Clínica Médica.

A busca bibliográfica foi desenvolvida nos sites oficiais das instituições referenciadas e nas bases de dados eletrônicas da Biblioteca Virtual em Saúde (BVS) - Literatura LatinoAmericana e do Caribe em Ciências da Saúde (LILACS) e Google Acadêmico. Foram utilizados os seguintes critérios de inclusão: artigos originais e disponíveis na íntegra em formato eletrônico em inglês e/ ou espanhol e/ou português, nos quais deveriam constar como assunto principal: cuidados paliativos", "cuidados paliativos em ambiente hospitalar". Como critérios de exclusão consideraram-se indisponibilidade na íntegra, duplicidade e o não atendimento ao objeto de estudo. 


\section{DISCUSSÃO E RESULTADOS}

Este relato é baseado na experiência que vivenciei como médica residente em um dos Hospitais da cidade de Anápolis, durante o primeiro e segundo ano de residência na área de Clínica Médica. O Hospital é composto por cinco enfermarias, onde presta atendimentos á pacientes portadores de diversas comorbidades, sendo uma das referências da cidade em pacientes oncológicos, os quais em sua grande maioria possuem prognóstico reservado.

No Brasil, o câncer se encontra como a segunda causa de morte por doença, sendo então um problema de saúde importante, com altas taxas de incidência. Uma doença que gera uma situação de desamparo aos que enfrentam essa trajetória, compreendendo-se que por ser uma doença de caráter crônico evolutivo, pode ocasionar finitude e morte. ${ }^{7}$ Assim é notável a importância da paliação e seus cuidados ao paciente em sua fase terminal de vida, principalmente, a esse paciente oncológico, visando proporcionar um tratamento que tenha como principal objetivo a promoção do cuidar humanizado; dirigindo seu foco para alívio das necessidades espirituais e biopsicossociais, integrando crenças, práticas religiosas, culturais não somente do paciente, como dos familiares. ${ }^{8}$

Durante o estágio nessa unidade de medicina interna, pude observar que havia naqueles setores muitos pacientes em estado terminal, recebendo apenas cuidados de conforto, sedação e higiene; idosos debilitados, jovens acamados e totalmente dependentes de terceiros.

Existem mais de 7.000 serviços de cuidados paliativos em mais de 90 países no mundo, sendo no Brasil apenas 40 serviços especializados nessa área. Contudo com o aumento no número de casos de pacientes com câncer avançado e dependente de cuidados paliativos observa-se a necessidade de tais cuidados em redes hospitalares, já que boa parte desse perfil de pacientes não possui condições físicas e socioeconômicas para internações domiciliares, sendo necessária a hospitalização como forma de controle de sintomas ou até mesmo devido à falta de cuidadores qualificados.

Nesse período me deparei com inúmeras vivências diante desse perfil de pacientes, lidei com pacientes já em diagnóstico de paliatividade desde o acompanhamento ambulatorial á pacientes em que a paliatividade foi definida no hospital, mas também, com pacientes extremamente graves com uma limitação de cura importante sem definição de paliatividade, e até mesmo pacientes os quais familiares não aceitavam essa definição.

Grande parte dos profissionais é treinada para fazer todo e qualquer possível para manter o paciente vivo, e acabam se vendo perplexos diante de um paciente com doença crônica em fase avançada, que já não responde a qualquer terapêutica curativa. No entanto é nesse momento que a atuação do profissional passa de curativa para medidas que 
proporcionem maior conforto possível para a pessoa viver em plenitude tanto quanto puder até o momento da sua morte. É uma resposta ativa aos problemas causados por uma doença prolongada, incurável e progressiva, combinando a ciência com o humanismo. Sendo assim, é fundamental que o profissional de saúde "moderno" consiga perceber e conhecer profundamente as doenças envolvidas e as possibilidades de intervenção, farmacológicas ou não farmacológicas. ${ }^{\text {10 }}$

Um alerta é feito pela OMS em que pessoas estão vivendo e morrendo sozinhas, cobertas de medo, com suas dores, sintomas físicos não controlados e as questões psicossociais e espirituais não atendidas. Sendo, então, nossa realidade atual, cuidados paliativos carentes ainda de políticas públicas para incluir essa forma de assistência num atendimento de alta pertinência e eficácia.

Durante todo o estágio na unidade nos deparamos com equipes de técnicas de enfermagem e enfermeiros, distribuídos entre as cinco enfermarias. Nessa distribuição de enfermarias nos deparamos com pacientes de diferentes áreas, seja da clínica médica, obstetrícia e ginecologia, ortopedia e traumatologia, cirurgia geral e subespecialidades cirúrgicas, oncológicos; e dentre tantos os pacientes em cuidados paliativos. É notável uma dificuldade em abordar esses pacientes, já que em sua grande maioria são vistos como pacientes sem condições de tratamento curativo, em que boa parte os vê como tratamento somente de controle de dor ou até mesmo controle de sintomas. Algumas vezes, vistos como o paciente que familiares levaram para somente ter sua "morte" em um ambiente hospitalar. Outras como o paciente que "não tem mais o que fazer".

Estudos apontam que dentre os profissionais de saúde, aqueles que ficam mais tempo com o paciente paliativo, principalmente os pacientes ao final da vida compete á equipe de enfermagem. Assim um cuidado de enfermagem paliativo consiste em gerar conforto, agir e reagir adequadamente frente ao paciente e seus familiares, valorizar seu sofrimento e suas conquistas mesmo que mínima. É gerar o alívio dos sintomas, ser flexível, ter objetivos de cuidado e principalmente reconhecer o paciente como ser humano único. ${ }^{\text {io }}$

Outra realidade presenciada seria em relação aos familiares de um paciente em cuidados paliativos e suas reações diante do prognóstico instituído, desde a aceitação até o processo de negação. Primeiramente temos familiares conscientes e entendedores do propósito dos cuidados paliativos e da aceitação sobre as limitações curativas em relação às doenças, os quais compreendem a necessidade de impor os $\mathrm{CP}$ ao ente querido e que cuidados ainda são instituídos aos mesmos, garantindo-lhes qualidade de vida até o momento da morte. 
No entanto lidamos com familiares os quais se encaixam no processo de negação, solicitando que sejam feitas medidas farmacológicas e clínicas para que então a "cura" seja encontrada, esses familiares durante o processo de vivência na unidade foram o maior desafio desde então. Lidamos em situações em que o próprio paciente deseja "descansar", ou seja, que os procedimentos e tratamentos instituídos em direção à cura sejam interrompidos em troca de procedimentos que lhe traga conforto e uma "morte" digna, e na outra ponta estão familiares em sua grande parte histéricos e lutando contra a própria vontade do paciente- um núcleo familiar adoecido por completo.

No tratamento paliativo o envolvimento familiar é primordial, já que possuem papel importante no crescimento e desenvolvimento dos indivíduos e na recuperação da saúde. De acordo com a OMS em relação ao efeito dos cuidados paliativos nos familiares consiste sobre conversar em relação aos cuidados de fim de vida e a percepção deles sobre a assistência nessa fase, demonstrando um fator protetor para o desenvolvimento de depressão e luto complicado ${ }^{\mathrm{II}}$.

A doença envolve também todo o grupo familiar, e o doente passa a ser o foco principal de atenção, e para cuidar a família desenvolve e cria várias formas de apoio e ajuda. E esse apoio e participação é uma forma de mostrar ao doente que ele não está sozinho para enfrentar as dificuldades. Sendo assim, esse grupo familiar necessita de um maior aporte, suporte e informações dos profissionais da área da saúde. Essa oferta maior e o suporte para os cuidadores familiares beneficiará o doente, os próprios cuidadores e toda a equipe de saúde. II

Durante o tratamento é preconizado que sejam desenvolvidas ações aos familiares visando a perspectiva de cuidar e para que as demandas deles sejam atendidas. É um sistema de saúde para seus membros por reunir um conjunto de valores, crenças, conhecimentos e práticas que funcionam como guia para a promoção, prevenção e tratamento da doença. A compreensão dos familiares junto a satisfação e a capacidade para participar nas decisões relacionadas ao cuidado do paciente, que em grande maioria não pode cuidar de si só, ajuda no momento de tomar decisões por ele no dia a dia de sua enfermidade.

É comum ma mistura de sentimentos por parte dos familiares, devido á complexidade da situação de saúde do paciente, em que projetam a expectativa que ele permaneça vivo, sendo importante, portanto, que o familiar entenda sobre a assistência paliativa, gerando uma melhor interação com o paciente, colaborando na prestação dos cuidados e na relação com a equipe. 


\section{Considerações Finais}

Vivemos diante de um numero cada vez mais crescente de doenças ameaçadoras da vida sejam elas, agudas ou crônicas, com ou sem possibilidade de reversão ou tratamentos curativos. Sendo assim, surgem os cuidados paliativos como forma de ver com mais respeito o paciente, seus sofrimentos e de seus familiares. O cuidar na visão de paliatividade consiste em reconhecer e responder às necessidades por meio de uma visão ampla e transdisciplinar.

Não existe um lugar específico para implementar os cuidados paliativos, o local indicado é onde o paciente estiver, onde necessitar de cuidados. E um grande número dos pacientes e familiares opta por "falecer" nos hospitais, onde os cuidados paliativos devem ser realizados.

Embora seja necessário valorizar o aspecto do cuidar quando o curar não é mais possível, há muita dificuldade para que esse cuidado ocorra. Há deficiência de recursos humanos e materiais e falta de preparo da equipe multiprofissional no aspecto técnico para lidar com a ansiedade e reações próprias do doente e família, bem como despreparo técnico para realizar os procedimentos que amenizam o sofrimento.

Sendo assim, é notado á necessidade de implementar cuidados paliativos em redes hospitalares juntamente á uma equipe multidisciplinar afim de garantir melhor qualidade de vida àqueles pacientes portadores de doenças incuráveis.

\section{Referências Bibliográficas}

I. HERMES, H.R.; LAMARCA, I.C.A. cuidados paliativos: uma abordagem a partir das categorias profissionais de saúde. 2577-2588. Rio de janeiro. 2013.

2. GOMES, A.L.Z; OTHERO, M.B. Cuidados Paliativos. Estud. av. vol.3o no.88 São Paulo Sept./Dec. 2016

3. SILVA, R.C.F, HORTALE, V.A. Cuidados paliativos oncológicos: elementos para o debate de diretrizes nesta área.Cad. Saúde Pública, Rio de Janeiro, 22(10):2055-2066, out, 2006.

4. BOEMER, M.R. Sobre cuidados paliativos. Rev. esc. enferm. USP vol.43 no.3 São Paulo Sept. 2009

5. Manual de cuidados paliativos. Ministério da saúde.

6. .FROSSARD, A.G.S; SILVA, E.C.S. Experiência da residência multiprofissional em serviço social e cuidados paliativos oncológicos. R. Katál., Florianópolis, v. 19, n. 2, p. 28r-288, jul./set. 2016 
7. FERNANDES, M. A, et al. Percepção dos enfermeiros sobre o significado dos cuidados paliativos em pacientes com câncer terminal. Ciênc. saúde coletiva. vol.18 no.9 Rio de Janeiro Sept. 2013

8. Cardoso, D.H. CUIDADOS PALIATIVOS NA ASSISTÊNCIA HOSPITALAR: A VIVÊNCIA DE UMA EQUIPE MULTIPROFISSIONAL. Texto contexto enferm. vol.22 no.4 Florianópolis Oct. /Dec. 2013

9. BURLÁ, C; Py, L.. Cuidados paliativos: ciência e proteção ao fim da vida. Cad. Saúde Pública. 30 (6) Jun 2014

ı. PIMENTA, C.A.M. Cuidados paliativos: uma nova especialidade do trabalho da enfermagem? Acta paul. enferm. vol.23 no.3 São Paulo May/June 2010.

II. FERREIRA, N.M.L, et. al. Cuidados paliativos e família. Rev. Ciênc. Méd., Campinas, ${ }_{17}(\mathrm{I}): 33-42$, jan/fev., 2008

I2. NUNES, M.G.S; RODRIGUES, B.M.R.D. Tratamento paliativo: perspectiva da família. Rev. enferm. UERJ, Rio de Janeiro, 2012 jul/set; 20(3):338-43.

13. MATSUMOTO, D. Y. Cuidados Paliativos: conceito, fundamentos e princípios. In: Academia Nacional de Cuidados Paliativos (org.). Manual de cuidados paliativos. Rio de Janeiro: Diagraphic, 2009. p. I4-I9. 